\title{
Acute ptosis as a presentation of preseptal cellulitis leading to cerebral abscess in a patient with uncontrolled diabetes
}

\author{
Nor Roziah Razali, Yao Mun Choo \\ Razali NR, Choo YM. Acute ptosis as a presentation of preseptal cellulitis leading to cerebral abscess in a patient with uncontrolled diabetes. \\ Malays Fam Physician. 2021;16(1);136-138. https://doi.org/10.51866/cr1010
}

\section{Keywords:}

ptosis, preseptal cellulitis,

cerebral abscess, diabetes

\section{Authors:}

\section{Choo Yao Mun}

(Corresponding author)

MBBS (Hons)(Monash), MRCPCH

(UK)

Associate Professor, Department of

Paediatrics, Faculty of Medicine

University of Malaya, Kuala Lumpur

Malaysia

Email: yaomun@um.edu.my

\section{Nor Roziah Razali}

$M D$

Department of Emergency Medicine University Malaya Medical Centre Kuala Lumpur, Malaysia

\section{Abstract}

Acute ptosis due to preseptal cellulitis requires urgent medical attention, as the infection can extend posteriorly into the orbit, leading to significant visual and cerebral complications. We report a case of a 58-year-old woman with uncontrolled diabetes mellitus presenting with acute ptosis due to preseptal cellulitis. After initial resolution of fever with intravenous amoxicillin-clavulanate, she experienced a seizure due to cerebral abscess a week later and was treated with intravenous ceftriaxone. Preseptal cellulitis is usually treated on an outpatient basis with oral antibiotics, as it rarely extends posteriorly to cause cerebral complications. We wish to highlight the importance of admitting patients with preseptal cellulitis in patients with uncontrolled diabetes for intravenous antibiotics due to the potential for visual and cerebral complications.

\section{Introduction}

Acute ptosis is a medical emergency requiring urgent assessment. Ptosis results from dysfunction of the muscles that raise the eyelid or their nerve supply (oculomotor nerve for the levator palpebrae superioris and sympathetic nerves for the superior tarsal muscle). Preseptal cellulitis is one of numerous causes of acute ptosis, which can lead to posterior extension of the infection, causing meningitis and cerebral abscess.

\section{Case Report}

We report a case of a 58-year-old female with uncontrolled diabetes mellitus complaining of 3-day history of right eye ptosis, fever, rhinitis, and diarrhea. The right eyelids were inflamed, but the pupils and extraocular movements of the eye were normal. Visual acuity, cranial nerves, and neurological examination were unremarkable. Blood sugar was $19.9 \mathrm{mmol} / \mathrm{L}$ with $\mathrm{HbAlC}$ of $12.2 \%$. She was treated with intravenous amoxicillin-clavulanate for preseptal cellulitis with resolution of fever, rhinitis, and diarrhea. However, ptosis persisted, and she developed seizures one week later with new onset of pyrexia.

Clinically, nystagmus and lip-smacking were observed. Lumbar puncture showed biochemical meningitis with sterile cultures. Computed tomography revealed preseptal fluid collection with subdural collection, leptomeningeal enhancement, and a rim-enhancing lesion over the right frontal region consistent with preseptal cellulitis leading to partially treated meningitis complicated by cerebral abscess. She was treated with intravenous ceftriaxone for 8 weeks with complete resolution.

\section{Discussion}

Acute ptosis can be caused by the aponeurosis of the levator muscle, nerve abnormalities,trauma,i nflammation, or lesions of the lid (e.g.,preseptal cellulitis) or orbit. $^{1}$ Preseptal cellulitis describes an infection of the eyelid in which superficial periorbital soft tissues anterior to the orbital septum are affected and the orbital structures posterior to the septum are not affected. ${ }^{2}$ Two basic mechanisms operate in the formation of preseptal cellulitis. The first involves spreading of a local infection to the eyelid. In this process, the source of infection is penetrating trauma around the eyes or an infection of the skin or subcutaneous tissue, such as acute dacryocystitis, hordeolum, infected chalazion, severe conjunctivitis, impetigo, and erysipelas. Other causes include eye surgery or insect bites. In the second mechanism, the septicity originates from the focus of the infection, which is far from the orbit. ${ }^{3}$

Preseptal cellulitis typically presents with eyelid edema and erythema. The extent of infection is superficial and does not normally extend posteriorly into the orbit in a mild infection. In contrast, orbital cellulitis is recognized to cause cerebral complications. Visual acuity pupillary reactions, extraocular motility, and intraocular 
pressures are normal. Periorbital tissues may become infected as a result of trauma (including insect bites) or primary bacteremia. Differential diagnoses of preseptal cellulitis includes orbital cellulitis, adenoviral keratoconjunctivitis, allergic conjunctivitis, contact dermatitis, hordeolum, Kawasaki's disease, idiopathic orbital inflammation, thyroid eye disease, dacryocystitis, and dacryoadenitis. The Chandler Classification of Orbital Complications staging system ${ }^{4}$ is as follows:

- Stage I - Preseptal cellulitis

- Stage II - Inflammatory orbital edema

- Stage III - Subperiosteal abscess

- Stage IV - Orbital abscess

- Stage V - Cavernous sinus thrombosis.

The most common organisms are Staphylococcus aureus, Staphylococcus epidermidis, Streptococcus species, and anaerobes, which are bacteria that usually cause upper respiratory tract infections and external eyelid infections. Multiple factors can cause preseptal cellulitis, such as ocular trauma, paranasal sinusitis, upper respiratory tract infection, acute or chronic otitis media, and dental origin. Various antibiotic regimens have been used for the treatment of preseptal cellulitis, such as ampicillin, clindamycin, cephalexin, cefuroxime, ampicillin with sulbactam, and chloramphenicol. Preseptal cellulitis is usually treated on an outpatient basis with oral antibiotics. However, in immunocompromised patients, such as those with uncontrolled diabetes mellitus, prompt treatment with intravenous antibiotics is recommended. ${ }^{5}$

Preseptal cellulitis requires accurate diagnosis, as infection in immunocompromised patients can progress posteriorly into the orbit, potentially leading to significant visual and cerebral complications. Most complications are thought to be minimal, with the infection involving only the eyelid and superficial periorbital soft tissues without affecting the orbital structures, especially

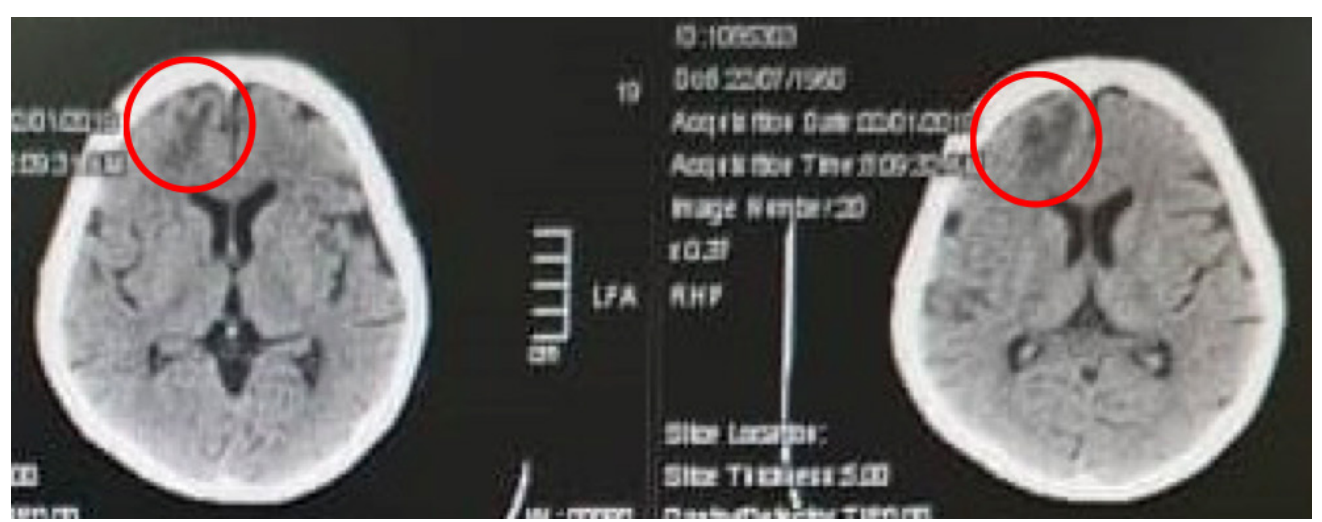

Figure 1: CT Brain showing preseptal fluid collection with subdural collection, leptomeningeal enhancement, and rim-enhancing lesion over the right frontal region if the condition is treated early during the course of infection. Prognosis is usually good with prompt diagnosis and treatment. However, complications can develop even with prompt treatment. Orbital extension and complications, such as orbital cellulitis, subperiosteal abscess, orbital abscess, or cavernous sinus thrombosis, can occur. ${ }^{6}$

Necrotizing fasciitis, a rare complication caused by $\beta$-hemolytic Streptococcus, can lead to necrosis and toxic shock syndrome. Central nervous system involvement with meningitis and cerebral abscess can occur after orbital extension. However, meningitis can occur even in the absence of significant clinical signs, leading to cerebral abscess.

Most case reports highlight septal cellulitis causing cerebral complications. In our case, this presentation of isolated unilateral ptosis was not typical of a neurogenic cause as the patient presented with preseptal cellulitis. Infections of the eyelids, nasolacrimal duct, conjunctiva, and corneal surface occur more frequently in people with uncontrolled diabetes. Administration of prompt intravenous antibiotics in immunocompromised patients, especially in the case of uncontrolled diabetes, is required in preseptal cellulitis due to the risk of hematogenous spread posteriorly, considering that some general practitioners may treat this condition on an outpatient basis with oral antibiotics. A CT scan is recommended in the case of marked eyelid swelling, fever, leukocytosis, suspected abscess, or no improvement after 24 hours on the proper antibiotics. ${ }^{4}$

In conclusion, acute ptosis caused by preseptal cellulitis in patients with uncontrolled diabetes mellitus requires diligent consideration of the possibility of intracranial infective extension causing meningitis and cerebral abscess. Cerebral abscess, though a rare complication of preseptal cellulitis, requires careful consideration in diabetic patients. 


\section{How does this paper make a difference to general practice?}

- This paper highlights the importance of recognizing preseptal cellulitis in a patient with uncontrolled diabetes and prioritizes administering intravenous antibiotics rather than oral antibiotics for immunocompromised patients.

- A CT scan is recommended in the case of marked eyelid swelling, fever, leukocytosis, suspected abscess, or no improvement after 24 hours on the proper antibiotics.

- Although septal cellulitis is known to cause cerebral complications, preseptal cellulitis can also lead to cerebral complications. Therefore, recognition of potential complications in an uncontrolled diabetic patient is important.

\section{References}

1. Carrie LM, David AC. Acquired ptosis: evaluation and management. American Academy of Ophthalmology [cited 10 August 2019]. Available from https://www.aao.org/ eyenet/article/acquired-ptosis-evaluationmanagement

2. Chaudhry IA,ShamsiFA. Outcome of treated orbital cellulitis in a tertiary eye care center in the Middle East. Ophthalmology. 2007;114(2):345-54.
3. Wald ER. Periorbital and orbital infections. Infect Dis Clin N Am. 2007;21(2):393-408.

4. Bae C, Bourget D. Periorbital cellulitis. [Updated 2020 Jul 21]. In: StatPearls. Treasure Island (FL): StatPearls Publishing; 2020. Available from: https://www.ncbi.nlm.nih.gov/ books/NBK470408.
5. Howe L., Jones NS. Guidelines for the management of periorbital cellulitis/abscess. Clin Otolaryngol. 2004;29:725-728.

6. Seongmu L,Michael T. Management of preseptal and orbital cellulitis. Saudi J Ophthalmol. 2011;25(1):21-29. 